\title{
25808
}

\section{Redefining broadband - effective spatial resolution}

\author{
M. Francis (Schlumberger), C. Cunnell (Schlumberger), S. Hydal \\ (Schlumberger), M. Tham (Schlumberger) \& M. Bayly* (Schlumberger)
}

\section{SUMMARY}

The positive impact of broadband acquisition and processing methods on the interpretation of seismic volumes has been well documented in recent years. While most gains have been achieved by extending low-frequency content, potential pitfalls exist for interpreters at both ends of the frequency spectrum, and care must be taken to understand the effective bandwidth of data. In addition, the handling of spatial frequencies (or wavenumbers) is as important as considerations for temporal aliasing. A complex earth creates a complex seismic wavefield in all directions, and so demands a revised definition of broadband with an emphasis on spatial resolution. We contend that the time has come to adopt the concept of effective spatial broadband based on high-resolution interpretation independent of orientation, and move towards the next level in geological understanding from our seismic volumes. 


\section{Introduction}

Broadband acquisition and processing techniques have positively impacted our ability to interpret seismic image volumes. A narrower wavelet gives sharper resolution and the reduction in side-lobe energy leads to a more direct view of the geology. This improves our ability to assess risk on stratigraphic traps. As interpreters, we must take care that the frequency content observed in the data is real. Frequency spectra contain both signal and noise. It is often difficult for geophysicists to measure a reliable signal-to-noise ratio on the final migrated section. Methods used to measure noise can become the basis of methods to suppress the noise. As a result, the residual noise becomes ever closer to resembling signal. We are also learning that much of the noise comes from the spatial sampling related to acquisition. For example, shallow overburden noise will alias and appears at different spatial frequency. We require adequate sampling to avoid such spatial aliasing, and so survey design is influenced as much by noise characterization as by signal requirements.

\section{Redefining broadband}

The Earth presents complex 3D geology, so we must consider what happens to our signal in such environments. Complexity in the seismic wavefield results from large-scale macrostructures as well as distortions caused by smaller geologic features located closer to the source and receivers. Our assumptions about emergent waves propagating vertically no longer apply. We must expand our definition of broadband to encompass spatial resolution in all directions (Figure 1). This places new demands on the methods required to sample and reconstruct the seismic wavefield, and a greater emphasis on controlling both amplitude and phase across the full bandwidth. 'Effective bandwidth' relates to our ability to mould our seismic data to any model of bandwidth we choose. A model can extend, contract, or shape the apparent bandwidth to meet each individual interpreter's objectives or preference. However, the input data may contain information over a limited range of frequencies and so it is the signal-tonoise ratio that provides flexibility in the

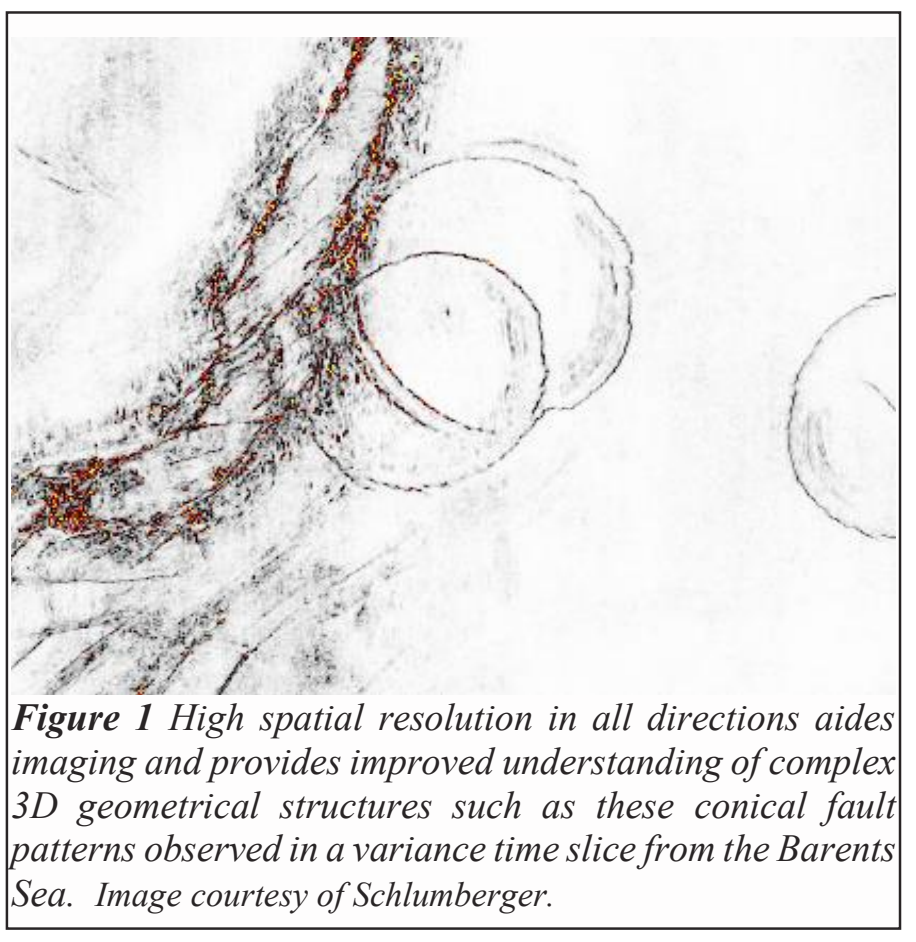
shaping process and maximizes the value of information at each frequency. This, in turn, relies on the ability to characterize and remove noise.

\section{Conclusions}

In this paper we present the concept of effective spatial broadband, where the criteria for broadband measurements includes the ability to provide high-resolution images of geological features irrespective of their orientation in the earth. This is complemented by the range of wavenumbers (in $x, y$, and $z$ ) over which useful information content contributes to the image volume. As interpreters, we welcome new insights gained from seismic data as they lead to more discrimination of lithology and rock properties, but we must recognize that this, in turn, makes demands on the data fidelity.

\section{Acknowledgements}

We thank Schlumberger for permission to publish these results. 